\title{
Research on Application of SNA in Tacit Knowledge Management of Project Team ${ }^{1}$
}

\author{
Xiao-hong Zhou ${ }^{1, a}$, Liang Wang ${ }^{1, b}$, Qing Cheng ${ }^{2, c}$ \\ ${ }^{1}$ Anhui Polytechnic University, Wuhu, Anhui Province, China \\ ${ }^{2}$ Hohai University, Nanjing, Jiangsu Province, China \\ azxh@ahpu.edu.cn, b2005022127@163.com, c1536802582@qq.com
}

\section{Key words: Project; Tacit knowledge; Social Network Analysis}

\begin{abstract}
The acquiring and sharing of tacit knowledge depend on social network mostly, as tacit knowledge of project team refers to collaboration and communication, knowledge management based on IT can't manage it effectively. On the basis of introduction of Social Network Analysis (SNA), this paper applies it into tacit knowledge management. The result indicates that SNA can describe network structure of tacit knowledge of project team, discover problems which cumber tacit knowledge management, and promote tacit knowledge management of project team.
\end{abstract}

\section{Introduction}

In the process of management, besides common explicit knowledge, the other category is tacit knowledge which consists of experience, skill and insight with no words to express. Tacit knowledge management relates to human behaviors. At present, studies about tacit knowledge management are mostly from the aspects of organization study and knowledge innovation, but lack quantitative analysis. As Social Network Analysis (SNA) grows and matures, scholars start to use SNA to study tacit knowledge management of intra-organization, it can reflect and quantify social relationships of members in organization as an important way to diffuse tacit knowledge which makes implicit information and knowledge clear. This article applies SNA into project management and analyzes tacit knowledge management of the project team.

\section{Social Network Analysis and tacit knowledge management}

SNA is a specific tool of Social Network Theory to deal with relationships, information or knowledge between entities mainly like people, groups, organizations and assess their value ${ }^{[1,2]}$. Based on mutual influence, the analysis from theoretical perspective focuses on relationships between actors, rather than their characteristics ${ }^{[3]}$. Social network is composed of a collection of nodes (actors) and connections among nodes (relations) which are used to indicate the network, so a good formal definition of SNA is obtained. The node could be a person or group, the connection means relationship or mutual flow direction between nodes.

Project team knowledge is experience, values, relevant information and insight of team members which is originated from every member's mind and act on them, project team can't get knowledge if depart from people. Moreover, a large part of knowledge is obtained when contacting with others rather than formative text materials. The characteristics of tacit knowledge dissemination is that each one's knowledge is closely related to ones they know which means people get information, knowledge and approach to problems mostly depend on personal social relationship network ${ }^{[3]}$. Whether an organization achieves complete exchange and sharing of knowledge lies on the strength of links between members. People and connections between them make the invisible network of knowledge management ${ }^{[2]}$ 。

From the organization point of view, first of all, as a result of trust among members in the internal relationships network, it reduces complexity of organization and speeds up communication and feedback of knowledge and information; then, success of network promotes not only creation and transfer of knowledge in organization, but also combination and exchange of resources ${ }^{[4]}$.In 
social network, a single organization makes a network node and gets connection relying on links. Information flows through links and network ${ }^{[5]}$. Therefore, Social Network is considered to be the best channel to transfer tacit knowledge, Applications of Social Networking will help to promote the socialization of knowledge creation, transfer, sharing and utilization ${ }^{[6]}$.Currently, the most difficult problem is a lack of effective tools and methods in organization knowledge management especially in tacit knowledge. SNA provides a theoretical and methodological guidance for tacit knowledge sharing in knowledge management from the point of invisible network ${ }^{[2]}$.

\section{Social network analysis steps of tacit knowledge management of the project team}

The SNA indicators of tacit knowledge management in project team include network density, average distance, network roles, network location, centrality, and cliques, etc ${ }^{[7]}$. The steps of SNA to promote the knowledge management of the project team are as follows:

(1) Set objectives: Set a target to know what you get from the social network. For example, reveal how to communicate with each other in different groups about knowledge.

(2) Collect data: This is mainly through tracking and observation of the actual situation (such as E-mail, daily records, internal instant messaging, etc.), questionnaires, and interviews to get relevant data of information flow, knowledge transfer mode.

(3) Draw net structure: Use SNA software (UCINET and so on) to get preliminary social network picture by transferring investigatory data to relationship data between nodes.

(4) Network structure analysis: according to above indicators, this paper explores various problems in information flow and knowledge sharing of individual, team and organization.

(5) Explain results of analysis: Through interview and deep research to explain further reasons of problems above.

(6) Interpose and evaluate the intervention: The study seeks to promote dissemination of knowledge and innovation according to reasons above. After a certain period of time, by observing information flow and changes of knowledge in communication network, this study evaluates the effectiveness of the intervention.

\section{Application of SNA in tacit knowledge management}

In order to show application of SNA in tacit knowledge management of project team, a R\&D project case was conducted.

Network Structure

Here forms communication network by measuring frequency of links between actors (as shown in Figure 1).

The average density of the network is 0.9091 , this numerical value is relatively moderate. At the same time, by further calculation, this study finds that there is reachability between any two actors, so even if they do not have a direct link, they can find each other through media.

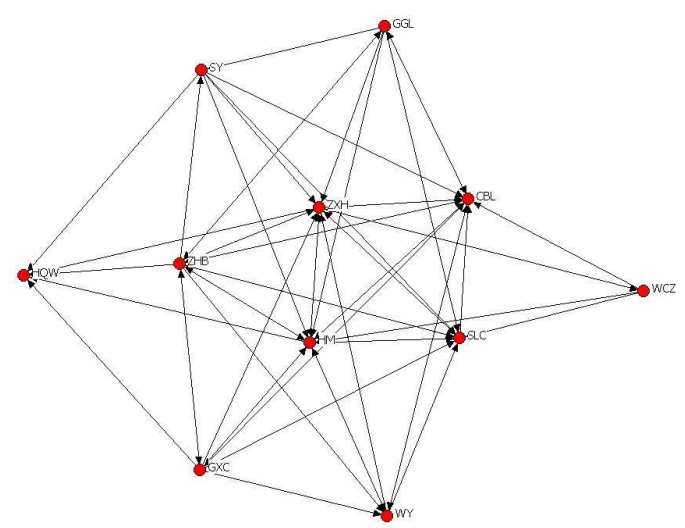

Figure 1 Communication network 
The network average distance is 1.470 , that is, one actor can contact with another by media of less than one. As is shown in Table 2, the longest distances are 5 from CBL to GGL and from CBL to GXC. If CBL would like to find GGL, or CBL want to find GGL, they both need another 4 individual as the media, the common cross-point is $\mathrm{ZZH}$, which reflects the role and the center position of him. Overall, the project team's communication network density is relatively moderate, longer distances appear in sub-groups which have little effect for the entire project. It may be appropriate to strengthen communication among sub-group leaders.

\section{Network Centrality}

As is shown in Table 1, centrality of CBL is in a high level of $13.55 \%$, the second is ZHB of $12.617 \%$. The whole network's centrality is $11.34 \%$. In comprehension, the centrality of advisory network is also moderate, key members represented by ZHD and CBL play a positive role in organization and promote knowledge transfer. However, the centrality of WCZ and HQW are low and become edge people of the network. They should enhance communication with the team.

Table 1 Advisory Network Centrality

\begin{tabular}{llll}
\hline & & Betweenness & nBetweenness \\
\hline 1 & CBL & 13.550 & 15.056 \\
10 & ZHB & 12.617 & 14.019 \\
5 & ZZH & 10.733 & 11.926 \\
7 & HM & 5.667 & 6.296 \\
2 & GGL & 1.500 & 1.667 \\
4 & SLC & 1.150 & 1.278 \\
9 & GXC & 0.933 & 1.037 \\
8 & WY & 0.600 & 0.667 \\
3 & SY & 0.250 & 0.278 \\
6 & WCZ & 0.000 & 0.000 \\
11 & HQW & 0.000 & 0.000 \\
\hline
\end{tabular}

\section{Network cliques}

From the Figure 2, WY, ZZH, HQW, SLC and SY to form a clique; ZZH, CBL, GXC, HM and ZHB form another; WCZ and GGL are apart. In fact, the research area and interest of GGL and WCZ are different from others, so they don't belong to any cliques in the knowledge network; WY, ZZH, HQW, SLC and SY, ZZH, CBL, GXC, HM and ZHB are in two sub-groups under a head group, they often discuss together which means great similarity. At the same time, as a bridge between two cliques, the positive role of ZZH brings possibility of cooperation between two cliques, and he should promote further transfer between two cliques. In addition, CBL and WY have a strong relationship in communication network, information network and knowledge network. Therefore, they should be encouraged to become the center of two cliques in order to improve knowledge transfer and innovation efficiency of project team ${ }^{[3]}$.

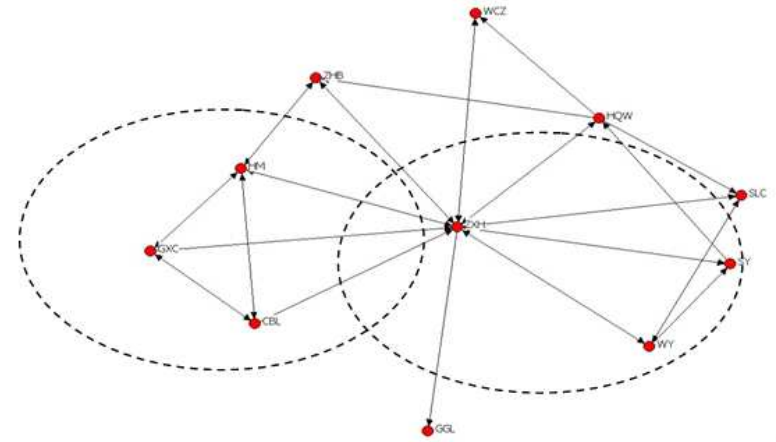

Figure 2 Project team knowledge network 


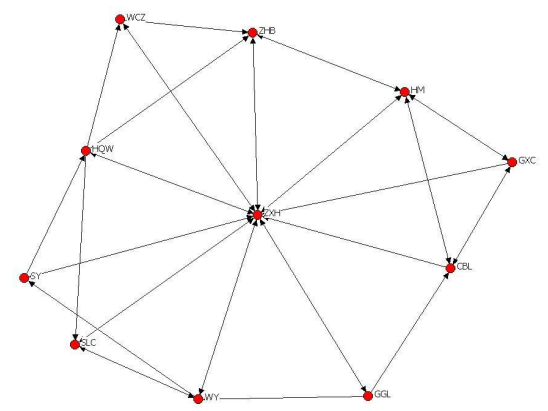

Figure 3 Project team knowledge network after two months

The diagram is also useful in description of network's change over time ${ }^{[8,9]}$. This study take some interference to project team against the situation in Figure 2, Figure 3 is the result, the former groups disappear, WCZ and GGL gradually integrate into the project team which means that project knowledge network changes over time.

\section{Conclusion}

To sum up, we can see that tacit knowledge of project team is a dynamic combination of members' experience, values, relevant information and insight, project members obtain and share tacit knowledge mostly depend on personal social relation network. The successful development of inner relation network promotes the creation and transfer of tacit knowledge in organization and gives impetus to a combination and exchange of resources. Social network is the best method to transfer tacit knowledge. SNA can quantify relation network of project organization in formalization and make quantitative analysis. Therefore, SNA can depict network structure of knowledge transfer in project team, get the root of problem about impeding transfer and sharing of tacit knowledge by quantitative analysis on network structure, and provide a reliable basis for formulating systems and measures of promoting tacit knowledge management.

${ }^{1}$ Research project of humanities and social science in Ministry of Education (Item number: 09YJE630001)

\section{References}

[1] Mead,S.P, Using Social Network Analysis to Visualize Project Teams, Project Management Journal (2001) 32-38.

[2] Wang Ping, Research on the tacit knowledge sharing based on the social network analysis, Information and Documentation Work, 2 (2006) 102-104.

[3] YIN Guopeng, MO Yunsheng, Chen Yu, Promoting tacit knowledge management using social network analysis, Journal of Tsinghua University (Science and Technology), 46 (2006) 964-969.

[4] Huang Xiaohua, Zhang Yang, Business knowledge management based on relation network, Science and Technology Management Research, 2 (2006)130-133.

[5] CHEN Di, An analysis of the social network on promoting organizations knowledge acquisition capacity, Journal of Southeast University (Philosophy and Social Science), 6 (2006) 84-88.

[6] Ma Feicheng, Wang Xiaoguang, The social network model of knowledge transfer, JX Social Sciences, 7 (2006) 38-44.

[7] Liu Jun, Introduction to the Social Network Analysis, Social science academic press (Beijing, China), 2004, pp. 83-183.

[8] Pryke,S.D, Analysing construction project coalition: exploring the application of social network analysis, Construction Management and Economics, 22(2004) 789-797.

[9] Pryke,S.D, Towards a social network theory of project governance, Construction Management and Economics, 23 (2005) 927-939. 